\title{
A versatile route to modify polyethersulfone membranes by chemical reduction of aryldiazonium salts
}

\author{
M. Picot, R. Rodulfo, I. Nicolas, A. Szymczyk ${ }^{*}$, F. Barrière*, M. Rabiller-Baudry \\ Université de Rennes 1, Institut des Sciences Chimiques de Rennes, UMR CNRS 6226, 263, \\ Avenue du Général Leclerc, 35042 Rennes cedex, France.
}

Corresponding authors:

*frederic.barriere@univ-rennes1.fr (F. Barrière)

*anthony.szymczyk@univ-rennes1.fr (A.Szymczyk)

\begin{abstract}
Ultrafiltration polyethersulfone membranes were modified covalently by chemical reduction of aryl diazonium salts. Functionalizations were performed with four aryl diazonium salts bearing different functional groups (4-benzyltriphenylphosphonium diazonium, 4-nitrophenyl diazonium, 4-benzonitrile diazonium and 4-phenylacetic acid diazonium) so as to demonstrate the versatility of the method. The efficiency of the different functionalizations was checked with various characterization techniques. Attenuated Total Reflectance Fourier Transform Infra Red spectroscopy revealed the presence of 4-nitrophenyl, 4-benzonitrile and 4phenylacetic acid groups at the surface of the different modified membranes but no characteristic vibration band was detected on the surface of the membrane modified with 4benzyltriphenylphosphonium diazonium. The presence of 4-benzyltriphenylphosphonium, however, could be demonstrated by both Energy Dispersive X-ray spectroscopy (detection of the $K \alpha$ ray of phosphorous at $2.015 \mathrm{keV}$ ) and streaming current measurements (shift of the membrane isoelectric point). Finally, dead-end filtration of an antibiotic (tylosin) was carried out with the unmodified membrane and the membrane modified by 4-benzyltriphenylphosphonium diazonium. Experiments revealed that the transport properties of the grafted membrane were significantly modified, with a significant increase in rejection mainly due to electrostatic repulsions between the surface of the modified membrane and tylosin.
\end{abstract}

Keywords: Ultrafiltration; Membrane functionalization; Covalent grafting; Aryldiazonium 


\section{Introduction}

The widespread technological and economical importance of membrane processes in various fields such as food industry, desalination, fuel cells, water treatment and chemistry, has been the driving force for the development of a large array of materials. These are very often composite membranes that rely on different polymers and additives. They are designed as multi-layer materials whose part that rules the separation (called the active layer) represents only a very small fraction of the total thickness of the membrane. Because of economical stakes, the detailed composition of commercial membranes is generally not provided and is protected by patents so that one can not rely entirely on their commercial chemical formulae. Among the large variety of membranes, polyethersulfone-based membranes are a widely spread class of membranes that have found large scale applications such as fractionation and purification of proteins in the dairy industry.

In order to confer or tune specific properties of membranes such as molecular weight cut-offs (MWCO), surface hydrophilicity/hydrophobicity, surface charge density or antifouling properties, various routes of membrane modification technique have been used such as coating, blending, chemical grafting or a combination of these methods $[1,2]$. Among recently proposed approaches, Razi et al. reported the functionalization of a polyethersulfone (PES) membrane with zwitterionic monomers by photografting. After surface modification, they noted an improvement of the antibiofouling efficiency [3]. The same kind of UV grafting procedure has been applied by Abuhabib et al. who modified two commercial nanofiltration membranes by combining two monomers: acrylic acid and ethylenediamine dihydrochloride. Using this treatment, they noted an improvement for salt rejection and fouling resistance [4]. Another example of surface modification has been reported by Rajesh et al. who described $\mathrm{TiO}_{2}$ nanoparticles impregnated PES membranes. The efficiency of the modified membrane in the separation of mixture solutions of divalent salt and surfactant were found to be improved significantly [5].

The chemical reduction of aryl diazonium salts has been rarely applied to membrane modification although polymer modification by aryl diazonium salts reduction has been known for some time [6]. To our knowledge only one study has reported modification of a cation exchange membrane using this technique [7]. Surface modification with aryl diazonium salt reduction is a versatile method since it allows the introduction of a large 
diversity of chemical groups at the surface so that many interfacial properties may be altered or tuned.

Modification of membrane surfaces is interesting not only for changing or optimizing some specific interfacial properties but it has also the potential to contribute to the deciphering of the membrane composition and to the knowledge of its physico-chemical properties through the analysis of changes brought about by the modification in terms of filtration performances, hydrophilicity/hydrophobicity, surface charge, etc.

The aim of this work is to demonstrate the feasibility of modifying PES filtration membranes by a simple and versatile method leading to robust grafting through covalent bonds. The versatility of the method was demonstrated by using aryl diazonium salts bearing different functional groups. The parent aryl-amines, namely 4-aminobenzyltriphenylphosphonium (1-

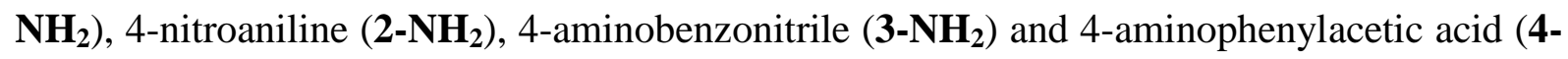
$\mathbf{N H}_{2}$ ) were used for the in situ generation of 4-benzyltriphenylphosphonium diazonium (1$\left.\mathbf{N}_{\mathbf{2}}{ }^{+}\right)$, 4-nitrophenyl diazonium $\left(\mathbf{2}-\mathbf{N}_{\mathbf{2}}{ }^{+}\right)$, 4-benzonitrile diazonium $\left(\mathbf{3}-\mathbf{N}_{\mathbf{2}}{ }^{+}\right)$and 4-phenylacetic acid diazonium $\left(4-\mathbf{N}_{2}{ }^{+}\right.$) salts, respectively. These chemical groups were selected to illustrate the versatility of the method since they possess different hydrophilic/hydrophobic features as well as different charge properties (uncharged, negatively or positively charged). Several characterization techniques were implemented to put in evidence the efficiency of membrane functionalizations. Finally, the impact of the chemical modification on transport properties was highlighted by comparing the experimental rejection of an antibacterial agent (tylosin) by the PES membrane before and after modification by $\mathbf{1 - \mathbf { N } _ { 2 }}{ }^{+}$.

\section{Materials and Methods}

\subsection{Chemicals and membrane modification procedure}

4-Nitroaniline (Fluka), 4-aminobenzonitrile (Acros Organics), 98\% 4-aminophenylacetic acid, 98\% (Acros Organics), sodium nitrite $97 \%$ and hypophosphorous acid $\left(\mathrm{H}_{3} \mathrm{PO}_{2}, 50 \mathrm{wt} \%\right.$ in water, Aldrich) were used as received. (4-Aminobenzyl)-triphenylphosphonium bromide, was obtained by the reduction of the commercial (4-nitrobenzyl)-triphenylphosphonium bromide $[8,9]$. 
Tylosin tartrate (Sigma) was dissolved in water to perform ultrafiltration experiments. A 0.1 $\mathrm{M} \mathrm{HCl}$ solution was used to adjust the $\mathrm{pH}$.

Ultrafiltration PES membranes (HFK-131, Koch Membrane Systems) with a Molecular Weight Cut-Off (MWCO) in the range 5-10 kD were used. According to manufacturer specifications, the maximum operating pressure of HFK-131 membranes is 9.7 bar, their operating temperature range is $5-55^{\circ} \mathrm{C}$ and the range of allowable $\mathrm{pH}$ for continuous operations is $2-10$.

HFK-131 membranes were first washed using ultra pure water (milli-Q quality) and were sonicated ( 2 x 20 minutes) in order to remove preservatives [10].

PES membranes were modified covalently using the chemical reduction of aryl diazonium salts [11-13]. The following experimental procedure was used. Aryl diazonium salts were generated in situ in acid media $(0.1 \mathrm{M} \mathrm{HCl})$ containing $50 \mathrm{mM}$ of the starting aryl amine and followed by addition of sodium nitrite at $100 \mathrm{mM}$ [11,12] (the chemical structures of the various aryl diazonium salts are shown in Fig. 1a). Immediately after that, the chemical reductant $\left(\mathrm{H}_{3} \mathrm{PO}_{2}\right)$ was also added in the solution $(10 \mathrm{~mL})$ in order to reduce the in situ generated aryl diazonium salt and form the corresponding aryl radical.Then, PES membranes samples (roughly $10 \times 10 \mathrm{~cm}$ ) were dipped in the reactive solution and left to react overnight. The proposed mechanism of grafting [6,7] onto the PES membrane (Fig. 1b-d) proceeds through radical attack on the polymer unsaturations, preferentially on the more electron rich carbon sites of the polarized phenyl rings (para-substituted with electron-donating ethers and electron-withdrawing sulfones). After modification, membranes were rinsed using ultra pure water and sonicated for $2 \times 20$ minutes in order to remove absorbed or loosely bound material from their surfaces (like polyphenylene oligomers). The advantages of this modification method are its ease of implementation, its versatility in terms of chemical functionalities and its strong and solvent resistant covalent grafting.

\subsection{Attenuated Total Reflectance-Fourier Transform Infra Red Spectroscopy (ATR-FTIR)}

IR spectra were recorded on a Brüker Optics Vertex 70 spectrometer using a diamond crystal ATR (Pike) and a liquid $\mathrm{N}_{2}$-cooled Mercury-Cadmium-Telluride (MCT) detector. All spectra (100 scans at $4 \mathrm{~cm}^{-1}$ resolution) were recorded at $25 \pm 1^{\circ} \mathrm{C}$. 


\subsection{Energy Dispersive X-ray Spectroscopy (EDX)}

EDX experiments (JEOL JSM 6400, OXFORD detector, INCA system) were conducted

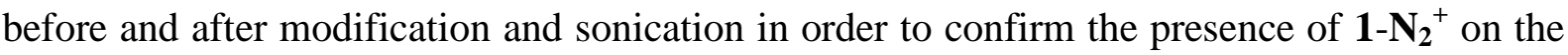
modified membrane (since 1- $\mathbf{N}_{\mathbf{2}}^{+}$was not detectable by ATR-FTIR; see below).

\subsection{Streaming current measurements}

Surface charge properties have a significant influence on membrane performances (rejection of charged solutes, fouling, etc.) and electrokinetic measurements are attractive methods of surface charge characterization of both polymeric $[14,15]$ and ceramic [16] membrane materials.

In this work, we employed the tangential streaming current method to characterize the electrokinetic charge density of unmodified and modified PES membranes. When an electrolyte solution is forced through a channel formed by two macroscopic surfaces facing each other, the fluid moves tangentially to the channel walls, pulling the ions towards the lowpressure side. In the case of charged materials, the electrical double layers that develop in the vicinity of the solid surfaces contain an excess of counterions that balance the surface charge. The convective transport of the excess of (hydrodynamically mobile) counterions in the direction of the liquid flow can be detected by measuring the electrical current (called the streaming current) between two up- and downstream positions via non-polarizable electrodes by an electrometer of sufficiently low internal resistance to make the back (conduction) current through the channel negligible [17].

Experiments were performed according to the following procedure. Two identical rectangular membrane samples were attached to the surfaces of the cell by means of double-sided adhesive tape. The cell used in this work was designed by CAD Instruments: it allows adjusting the channel height (i.e. the distance of separation between the membrane samples) with two micrometric screws. The channel height was determined from the cell hydraulic permeability using the Hagen-Poiseuille equation [18]. All experiments were performed with a channel height of $105 \pm 5 \mu \mathrm{m}$. Streaming current measurements were performed at $20 \pm 2$ ${ }^{\circ} \mathrm{C}$ with a ZetaCad electrokinetic analyzer (CAD Instruments). 
$0.001 \mathrm{M} \mathrm{KCl}$ solutions at various $\mathrm{pH}$ were used. A $0.1 \mathrm{M} \mathrm{HCl}$ solution was used to adjust $\mathrm{pH}$. The electrolyte solution was forced through the slit channel formed by the membrane samples using nitrogen gas. A differential pressure sensor was used to measure the pressure drop across the channel.

Prior to measurements, the solution was circulated through the channel for ca. 1 hour to allow for the sample equilibration. Streaming current was further measured by a pair of $\mathrm{Ag} / \mathrm{AgCl}$ electrodes, the equilibration process being monitored experimentally via the time dependence of the streaming current. After equilibration, the streaming current was measured and recorded for increasing pressure pulses ranging from 0 to $500 \mathrm{mbar}$, the flow direction being changed periodically. An example of experimental data is shown in Fig. 2 where the signs + and - for the pressure difference are associated with the different flow directions.

\subsection{Ultrafiltration experiments}

Dead-end filtration experiments were carried out with a stainless steel stirred cell. A circular membrane sample with an effective surface area of $43 \mathrm{~cm}^{2}$ was placed on the base of the cell which was filled with $300 \mathrm{~mL}$ of solution. The solution was stirred at $200 \mathrm{rpm}$ via a motorized stirring bar so as to limit concentration polarization. The stirred cell was pressurized with nitrogen gas and was equipped with a pressure relief valve.

Prior to filtration experiments, the membranes were compacted by applying a transmembrane pressure difference of 4 bar for 1 hour. After compaction, the pure water flux of membranes was measured as a function of the transmembrane pressure. Dead-end filtration experiments were then performed at 2 bar with a $1 \mathrm{~g} / \mathrm{L}$ tylosin tartrate solution. During experiments, permeate mass was measured as a function of time with an electronic balance and permeate samples were collected at regular intervals in order to determine solute concentration (tylosin concentration was determined by UV detection at $260 \mathrm{~nm})$. Tylosin rejection $\left(R_{\text {tylosin }}\right)$ was determined according to:

$$
R_{t y l o s i n}=1-\frac{C_{t y l o s i n}^{\text {perm }}}{C_{t y l o s i n}^{r e t}}
$$


where $C_{t y l o s i n}^{\text {perm }}$ and $C_{\text {tylosin }}^{\text {ret }}$ are tylosin concentrations in the permeate and retentate compartments, respectively.

Filtration was stopped when a volume reduction ratio (VRR) of around 2.5 was achieved. All experiments were performed at $20 \pm 2{ }^{\circ} \mathrm{C}$.

\section{Results and discussion}

\subsection{Physico-chemical characterization of membranes}

\subsubsection{Attenuated Total Reflectance - Fourier Transform Infra Red (ATR-FTIR)}

As received membranes exhibited a wide band around $3400 \mathrm{~cm}^{-1}$ which was assigned to the presence of preservatives in accordance with previous reports $[10,21]$. To remove these preservatives, the PES membranes were washed twice in an ultrasonic bath with ultrapure water for 20 minutes. Representative spectra of as received and washed PES membranes are shown in Fig. 3 and display the absence of the large band assigned to the preservatives after washing and drying of membranes. The spectrum of the washed PES membrane was found in good agreement with the literature [10, 19, 21] and notably displays two small bands associated with aromatic C-H vibration present at $3068 \mathrm{~cm}^{-1}$ and $3095 \mathrm{~cm}^{-1}$ (see inset of Fig. 3 ). We can also note the presence of typical bands at 1576 and $1480 \mathrm{~cm}^{-1}$ assigned to aromatic $\mathrm{C}=\mathrm{C}$ from the aryl ring.

Modified PES membranes were also characterized by ATR-FTIR. After modification by chemical reduction of aryldiazonium salts generating different radicals (4-nitrophenyl, 4benzonitrile and 4-phenylacetic acid), functionalized membranes exhibited characteristic bands. These bands, however, were considerably less intense than the bands arising from unmodified membrane (Fig. 4) but could nevertheless be assigned to the presence of the modifier on the surface. For the membrane modified with $3-\mathbf{N}_{2}^{+}$, a band at $2225 \mathrm{~cm}^{-1}(\mathrm{C} \equiv \mathrm{N}$ stretching band) was detected in an area clear of PES characteristic bands (Fig. 4a). In the case of the membrane modified with $\mathbf{2}-\mathbf{N}_{\mathbf{2}}{ }^{+}$, a characteristic band at $1520 \mathrm{~cm}^{-1}$ (asymmetric $\mathrm{NO}_{2}$ stretch) was identified between two intense PES characteristic bands (Fig. 4b). Finally, for the membrane modified with $4-\mathbf{N}_{2}{ }^{+}$, a band at $1725 \mathrm{~cm}^{-1}(\mathrm{C}=\mathrm{O}$ stretching vibration) was 
detected just at the footstep of the first intense PES membrane absorptions (Fig. 4c). This provides qualitative evidence that a robust grafting occurred on these membranes.

Despite the low intensity of their characteristic IR bands, these absorptions are not concealed beneath the intense PES membrane absorption and are luckily detectable in narrow windows almost free of signals arising from the unmodified membrane. We have hence found useful IR probes on the modifier that are compatible with the intense absorptions of the underlying PES membrane (Fig. 4).

For the last modifier, $\mathbf{1 - \mathbf { N } _ { 2 }}{ }^{+}$, it was not possible to use any of its characteristic bands to give evidence of an efficient grafting onto the PES membrane. Indeed, characteristic vibrations such as P-C (795-650 $\left.\mathrm{cm}^{-1}\right)$ and P-Aryl (1130-1090 and 750-680 $\left.\mathrm{cm}^{-1}\right)$ are not visible since the relatively intense IR bands of the unmodified PES membrane spectrum overlap with these vibrations.

\subsubsection{Energy Dispersive X-ray spectroscopy (EDX)}

Since the modification of the PES membrane by $\mathbf{1 - \mathbf { N } _ { \mathbf { 2 } }}{ }^{+}$was not detectable by ATR-FTIR, EDX spectroscopy was used to get elemental analysis of the membrane surface. As can be seen from Fig. 5, the $K \alpha$ ray $(2.015 \mathrm{keV})$ of the chemical element phosphorous $(\mathrm{P})$ was observed on the spectrum of the modified membrane, which gives evidence of the efficiency of membrane functionalization by 4-benzyltriphenylphosphonium. Control experiments have confirmed that the signal for $\mathrm{P}$ originates from the phosphonium modifier and not from $\mathrm{H}_{3} \mathrm{PO}_{2}$ contamination.

\subsubsection{Streaming current measurements}

In order to confirm the results of EDX experiments regarding the PES membrane modified using $\mathbf{1}-\mathbf{N}_{2}{ }^{+}$(bearing the positively charged phosphonium functionality), streaming current measurements were also carried out since this electrokinetic technique is sensitive to any change in the surface electrical properties of a macroscopic solid. As illustrated in Fig. 2, the streaming current varies linearly with the pressure drop through the channel. The slope is defined as the streaming current coefficient. Its sign and magnitude give information about the surface charge properties. It is illustrated in Fig. 6 which shows the variation of the 
streaming current coefficient with the $\mathrm{pH}$ of a $0.001 \mathrm{M} \mathrm{KCl}$ solution for the unmodified PES membrane and the membranes modified by $\mathbf{1}-\mathbf{N}_{2}{ }^{+}$. Electrokinetic measurements indicated that the unmodified PES membrane was negatively charged. As shown in Fig. 6, the membrane charge increases (in absolute value) with the $\mathrm{pH}$ of the solution and tends to level off for $\mathrm{pH}>\sim 6$, which suggests that weak acid functionalities are present at the surface of the unmodified membrane.

The streaming current method confirms the efficiency of the membrane modification with the positively charged 4-benzyltriphenylphosphonium group since the initial negative charge of the PES membrane is significantly lowered (in absolute value) after surface modification and a significant shift of the isoelectric point towards higher $\mathrm{pH}$ values (which is a signature of the presence of positively charged surface groups after membrane functionalization) is put in evidence. It is worth noting, however, that the overall charge of the modified PES membrane remains negative over a wide range of $\mathrm{pH}$ which suggests that the membrane surface is not totally covered after grafting.

\subsection{Transport properties}

In order to assess the impact of surface functionalization by aryl radicals on the transport properties of PES membranes, dead-end filtration experiments were performed with both unmodified and functionalized membranes. The selected functionalized membrane was that modified by $\mathbf{1}-\mathbf{N}_{2}{ }^{+}$. Fig. 7 shows the rejection rate of tylosin by both membranes as a function of the volume reduction ratio (VRR).

At $\mathrm{pH} 5.9$ the unmodified PES membrane was found to reject only $10 \%$ of tylosin approximately. This very low rejection is mainly due to the small size of tylosin (the molar mass of tylosin is $916 \mathrm{~g} \mathrm{~mol}^{-1}$ ) with respect to the MWCO of the HFK-131 membrane (5-10 $\mathrm{kD})$. At identical $\mathrm{pH}$, the rejection of tylosin by the modified membrane increases up to 22-25 $\%$ depending on the VRR value. Although grafting of 4-benzyltriphenylphosphonium radicals onto the pore surface reduces the effective pore size of the membrane, it is unlikely that steric hindrance be the only mechanism responsible for such an increase in the tylosin rejection by the modified membrane. This can be justified by comparing the hydraulic permeabilities of the different membranes. Fig. 8 shows the pure water flux of both membranes against the transmembrane pressure difference. As expected, the hydraulic permeability of the modified membrane is smaller than that of the unmodified membrane but the permeability ratio is found to be 0.82 . Assuming a Poiseuille's law for the pressure dependence of water flux and 
ideal cylindrical pores, the effective pore radius of the modified membrane would be reduced by less than $10 \%$ with respect to the original pore size (according to Poiseuille's law, the hydraulic permeability decreases with the square of the pore radius), which cannot explain the rise of rejection from 10 up to $25 \%$. It is likely that electrostatic repulsions between the positively charged 4-benzyltriphenylphosphonium groups (see Fig. 1a) and the protonated amine group of tylosin $\left(\mathrm{pK}_{\mathrm{a}}=7.73\right.$ [22] $)$ also contribute to tylosin rejection. As discussed in section 3.1.3, the membrane surface is not fully covered by 4-benzyltriphenylphosphonium groups and the overall charge density of the modified membrane remains negative at $\mathrm{pH} 5.9$ which limits the tylosin rejection. This interpretation is fully supported by additional measurements carried out at $\mathrm{pH} 3.5$, that is at a $\mathrm{pH}$ for which the overall charge density of the modified membrane is positive (see Fig. 6). As can be seen in Fig. 7, the rejection by the modified membrane increases to $\sim 50 \%$ at $\mathrm{pH} 3.5$, which is in line with the stronger repulsive interactions between the targeted solute and the membrane. It should be stressed that hydrolysis of tylosin occurs in acidic medium and leads to the release of mycarose (neutral sugar) and the formation of tylosin B (or desmycosin) [23]. However, hydrolysis of tylosin is thought to be quite slow in our conditions of $\mathrm{pH}$ and temperature [24]. To check this point, we performed the UV spectra of the freshly-prepared tylosin solutions and those of the same solutions at the end of filtration experiments and we did not observe any difference for both pH 5.9 and 3.5. Anyway, this point does not question our analysis based on the contribution of electrostatic interactions since tylosin $\mathrm{B}$ is smaller than tylosin and remains positively charged at $\mathrm{pH} 3.5$ (the $\mathrm{pK}_{\mathrm{a}}$ of tylosin $\mathrm{B}$ is 8.36 [22]).

The above results give evidence that transport properties of the original membrane have been strongly modified. The versatility of the functionalization method has been demonstrated in section 3.1 and the modification of PES membranes via chemical reduction of aryl diazonium salts appears as a promising method for tailoring the separation properties of mesoporous PES membranes. In addition to its versatility, the method also has the advantage of allowing a covalent grafting occurring spontaneously by simple dipping of the membrane into a solution of the diazonium salt. It can therefore be advantageous, in terms of stability, compared to other methods leading to non-covalent functionalization [25]. 


\section{Conclusion}

This study demonstrated that chemical reduction of aryl diazonium salts could be successfully applied to the covalent functionalization of ultrafiltration PES membranes with a significant impact on their separation properties.

This method has several advantages that make it very attractive. First, the method is easy to implement since grafting occurs spontaneously by simply dipping the membrane into a diazonium salt solution. The second advantage is that it leads to a robust modification of membranes since covalent bonds are formed between aryl radicals and the membrane surface. Finally, the method offers a wide range of possibilities in terms of functional groups that can be grafted. Depending of the functionality of the group(s) that aryl radicals bear, this method could therefore be applied to achieve various specific goals, e.g. more hydrophilic membranes (the main disadvantage of PES membranes is related to their relatively hydrophobic character), membranes with anti-biofouling properties, catalytic membranes...

\section{Acknowledgements}

This research is supported by funding from the European Union's Seventh Framework Programme FP7/2007-2013 under Grant Agreement No. 226532. M. P. and I. N. thank the European Union for studentship and post-doctoral fellowship, respectively. The authors thank Isabelle Péron and Francis Gouttefangeas for EDX experiments. Cyril Herrier is acknowledged for advices regarding IR experiments. The authors thank Cyril Poriel, Joëlle Rault Berthelot and David Delaunay for fruitful discussions and helpful suggestions.

\section{References}

[1] B. Van der Bruggen, Chemical Modification of Polyethersulfone Nanofiltration Membranes: A Review. J. Appl. Polym. Sci. 114 (2009) 630-642.

[2] N. Nady, M.C.R. Franssen, H. Zuilhof, M.S. Mohy Eldin, R. Boom, K Schroën, Modification methods for poly(arylsulfone) membranes : A mini-review focusing on surface modification. Desalination 275 (2011) 1-9. 
[3] F. Razi, I. Sawada, Y. Ohmukai, T. Maruyama, H. Matsuyama, The improvement of antibiofouling efficiency of polyethersulfone membrane by functionalization with zwitterionic monomers. J. Membrane Sci. 401-402 (2012) 292-299.

[4] A.A. Abuhabib, A.W. Mohammad, N. Hilal, R.A. Rahman, A.H. Shafie, Nanofiltration membrane modification by UV grafting for salt rejection and fouling resistance improvement for brackish water desalination. Desalination 295 (2012) 16-25.

[5] S. Rajesh, A.F. Ismail, D.R. Mohan, Structure-property interplay of Poly (amide-imide) and $\mathrm{TiO}_{2}$ nanoparticles impregnated Poly (ether-sulfone) asymmetric nanofiltration membranes. RSC Advances, 2012, doi:10.1039/C2RA20265D

[6] D. Bélanger, J. Pinson, Electrografting: a powerful method for surface modification. Chem. Soc. Rev. 40 (2011) 3995-4048.

[7] X.T. Le, P. Viel, P. Jégou, A. Garcia, T. Berthelot, T.H. Bui, S. Palacin, Diazoniuminduced anchoring process: an application to improve the monovalent selectivity of cation exchange membranes. J. Mater. Chem. 20 (2010) 3750-3757.

[8] F.D. Bellamy, K. Ou, Selective reduction of aromatic nitro compounds with stannous chloride in non acidic and non aqueous medium. Tetrahedron Lett. 25 (1984) 839-842.

[9] M. Picot, L. Lapinsonnière, M. Rothballer, F. Barrière, Graphite anode surface modification with controlled reduction of specific aryl diazonium salts for improved microbial fuel cells power output. Biosens. Bioelectron. 28 (2011) 181-188.

[10] S.X. Liu, J.T. Kim, S. Kim, Effect of polymer surface modification on polymer-protein interaction via hydrophilic polymer grafting. J. Food. Sci. 73 (2008) 143-150.

[11] J. Lyskawa, D. Bélanger, Direct modification of a gold electrode with aminophenyl groups by electrochemical reduction of in situ generated aminophenyl monodiazonium cations. Chem. Mater. 18 (2006) 4755-4763. 
[12] S. Baranton, D. Bélanger, Electrochemical derivatization of carbon surface by reduction of in situ generated diazonium cations. J. Phys. Chem. B. 109 (2005) 24401-24410.

[13] F. Barrière, A.J. Downard, Covalent Modification of Carbon Surfaces by NonElectrochemical Methods J. Sol. State Electrochem. 12 (2008) 1231-1244.

[14] M. Sbaï, A. Szymczyk, P. Fievet, A. Sorin, A. Vidonne, S. Pellet-Rostaing, A. FavreRéguillon, M. Lemaire, Influence of the Membrane Pore Conductance on Tangential Streaming Potential. Langmuir 19 (2003) 8867-8871.

[15] A.E. Yaroshchuk, T. Luxbacher, Interpretation of Electrokinetic Measurements with Porous Films: Role of Electric Conductance and Streaming Current within Porous Structure. Langmuir 26 (2010) 10882-10889.

[16] M. Sbaï, P. Fievet, A. Szymczyk, A. Vidonne, Determining the $\zeta$-potential of plane membranes from tangential streaming potential measurements: effect of the membrane body conductance. J. Membrane Sci. 226 (2003) 227-236.

[17] C. Werner, H. Körber, R. Zimmermann, S. Dukhin, J. Jacobasch, Extended Electrokinetic Characterization of Flat Solid Surfaces. J. Colloid Interface Sci. 208 (1998) 329-346.

[18] A. Szymczyk, N. Fatin-Rouge, P. Fievet, Tangential streaming potential as a tool in modeling of ion transport through nanoporous membranes. J. Colloid Interface Sci. 309 (2007) 245-252.

[19] A. Rahimpour, UV photo-grafting of hydrophilic monomers onto the surface of nanoporous PES membranes for improving surface properties. Desalination 265 (2011) 93-101.

[20] A. Razmjou, J. Mansouri, V. Chen, The effect of mechanical and chemical modification of $\mathrm{TiO}_{2}$ nanoparticles on the surface structure and fouling performance of PES ultrafiltration membranes. J. Membrane Sci. 378 (2010) 73-84. 
[21] S. Belfer, R. Fainchtain, Y. Purinson, O. Kedem, Surface characterization by FTIR-ATR spectroscopy of polyethersulfone membranes-unmodified, modifed and protein fouled. J. Membrane Sci. 172 (2000) 113-124.

[22] J.W. McFarland, C.M. Berger, S.A. Froshauer, S.F. Hayashi, S.J. Hecker, B.H. Jaynes, M.R. Jefson, B.J. Kamicker, C.A. Lipinski, K.M. Lundy, C.P. Reese, C.B. Vu, Quantitative Structure-Activity Relationships among Macrolide Antibacterial Agents: In Vitro and in Vivo Potency against Pasteurella multocida. J. Med. Chem. 40 (1997) 1340-1346.

[23] R.A. Fernández, M.I. Velasco, L.I. Rossi, S.A. Dassie, Transfer of tylosin across the $\mathrm{H}_{2} \mathrm{O} / 1$,2-dichloroethane interface - Analysis of degraded product in acid solutions. J. Electroanal. Chem. 650 (2010) 47-54.

[24] J. Paesen, W. Cypers, K. Pauwels, E. Roets, J. Hoogmartens, Study of the stability of tylosin A in aqueous solutions. J. Pharm. Biomed. Anal. 13 (1995) 1153-1159.

[25] A. Keraani, M. Rabiller-Baudry, C. Fischmeister, C. Bruneau, Immobilisation of an ionically tagged Hoveyda catalyst on a supported ionic liquid membrane: an innovative approach for metathesis reactions in a catalytic membrane reactor. Catalysis Today 156 (2010) 268-275. 
a)<smiles>N#[N+]c1ccc(CP(c2ccccc2)c2ccccc2)cc1</smiles>

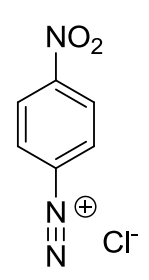

$2-\mathrm{N}_{2}^{+}$

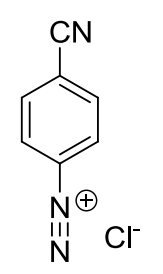

3- $\mathrm{N}_{2}^{+}$

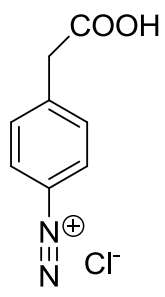

4- $\mathbf{N}_{2}{ }^{+}$

b) $\mathrm{R} \longrightarrow \mathrm{NH}_{2} \stackrel{\mathrm{NaNO}_{2}+\mathrm{HCl}}{0^{\circ} \mathrm{C}}$<smiles></smiles>

1- $\mathrm{NH}_{2} \mathrm{R}=\mathrm{CH}_{2} \mathrm{P}^{+} \mathrm{Ph}_{3}$

2- $\mathrm{NH}_{2} \mathrm{R}=\mathrm{NO}_{2}$

Aryldiazonium salt

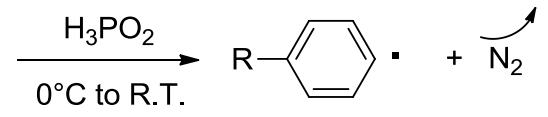

Aryl radical

3- $\mathrm{NH}_{2} \mathbf{R}=\mathrm{CN}$

4- $\mathrm{NH}_{\mathbf{2}} \mathbf{R}=\mathrm{COOH}$

c)<smiles>[R]c1ccccc1</smiles>

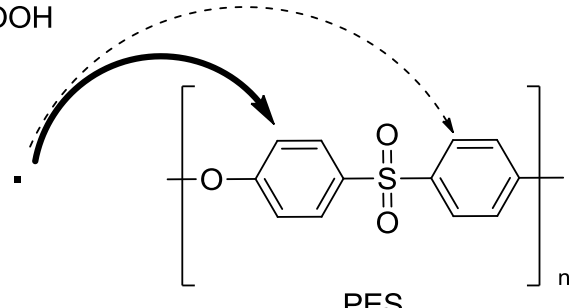
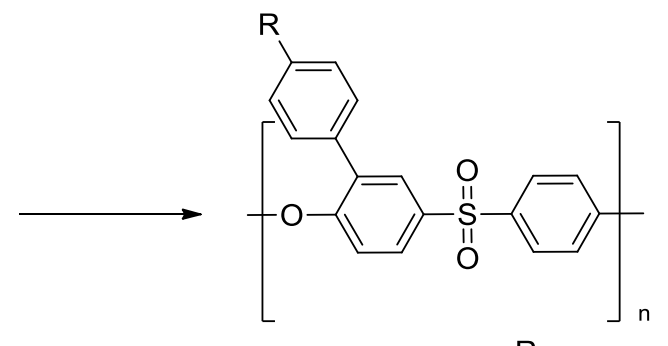

PES

d)<smiles>[R]c1ccc(CC)cc1</smiles><smiles>[R]c1ccc(-c2ccccc2OCC)cc1</smiles><smiles>CCCCCCS(=O)(=O)c1ccc(C)cc1</smiles><smiles>C#CC</smiles><smiles>[R]c1ccc(-c2cc(-c3cc(OC)c([R])c(-c4ccc([R])cc4)c3)ccc2S(=O)(=O)c2ccc(CC(C)(C)C)cc2)cc1</smiles>

Figure 1. (a) Chemical structure of in situ-generated aryl diazonium salts. From left to right:

4-benzyltriphenylphosphonium diazonium $\left(\mathbf{1}-\mathbf{N}_{\mathbf{2}}{ }^{+}\right)$, 4-nitrophenyl diazonium $\left(\mathbf{2}-\mathbf{N}_{\mathbf{2}}{ }^{+}\right), 4-$ benzonitrile diazonium $\left(3-\mathbf{N}_{2}{ }^{+}\right)$and 4-phenylacetic acid diazonium $\left(\mathbf{4 - \mathbf { N } _ { 2 }}{ }^{+}\right)$. (b-d) Proposed mechanism for Polyethersulfone (PES) modification through aryl diazonium salt reduction using hypophosphorous acid as a reducing agent. 


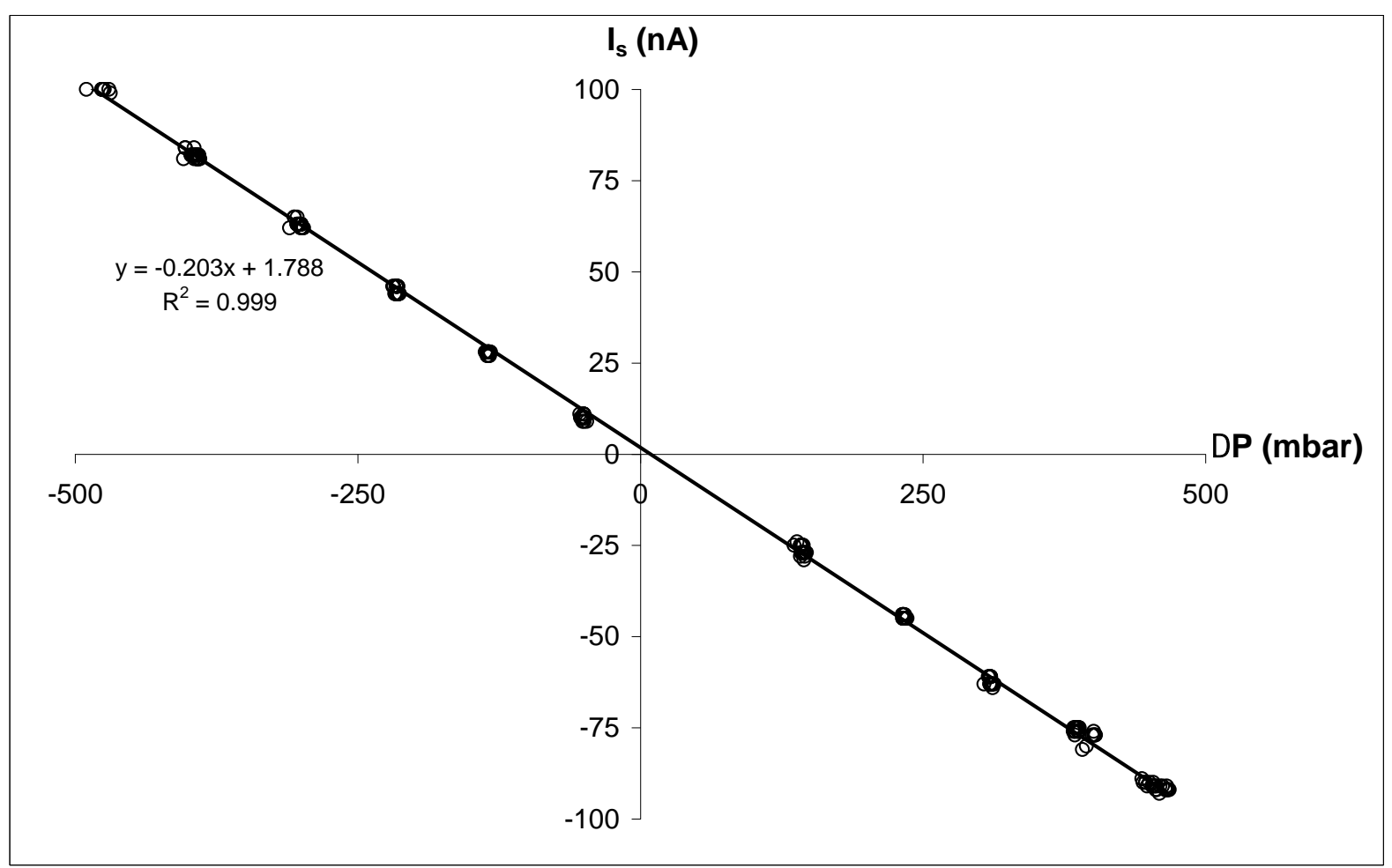

Figure 2: Streaming current $\left(\mathrm{I}_{\mathrm{s}}\right)$ vs. pressure difference $(\Delta \mathrm{P})$; Unmodified PES membranes in $10^{-3} \mathrm{M} \mathrm{KCl}$ solution at $\mathrm{pH} 5.4$. 


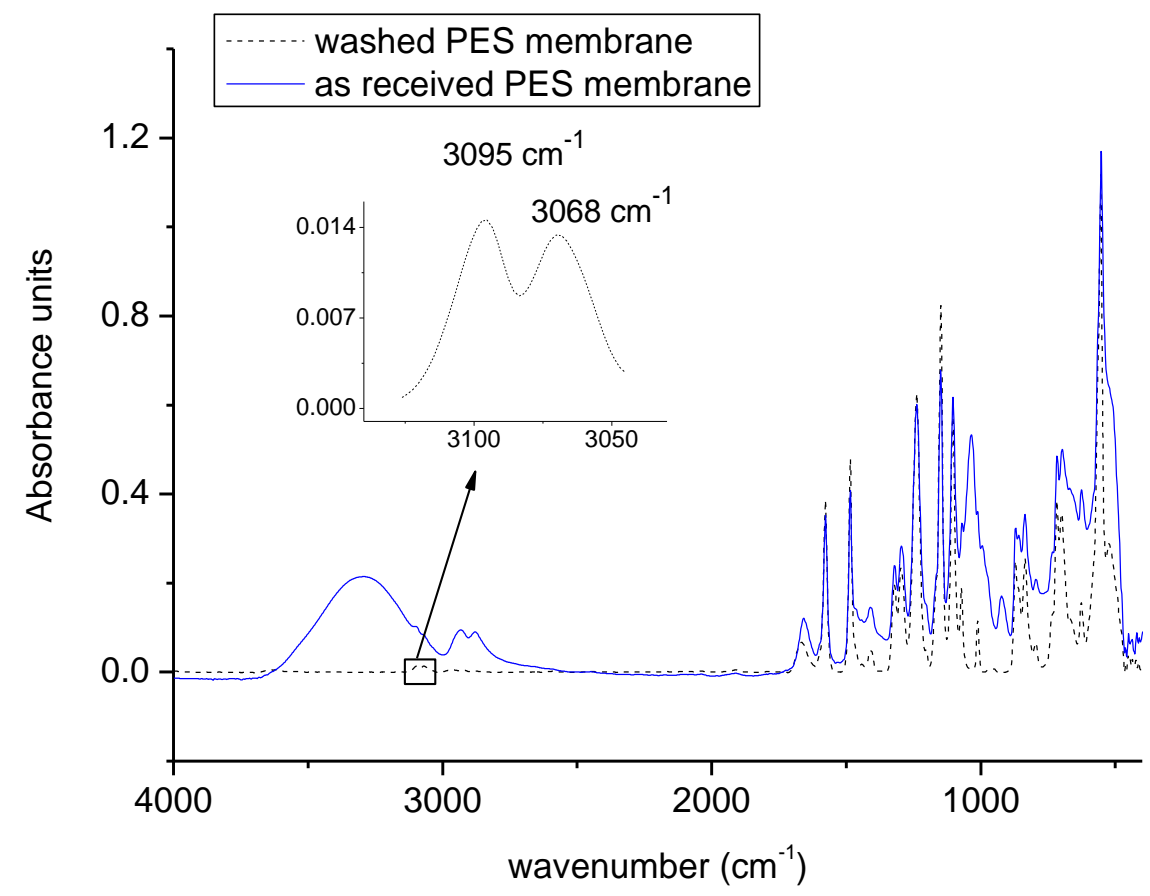

Figure 3: Representative ATR-FTIR spectra of as received and of washed and dried PES membranes. 


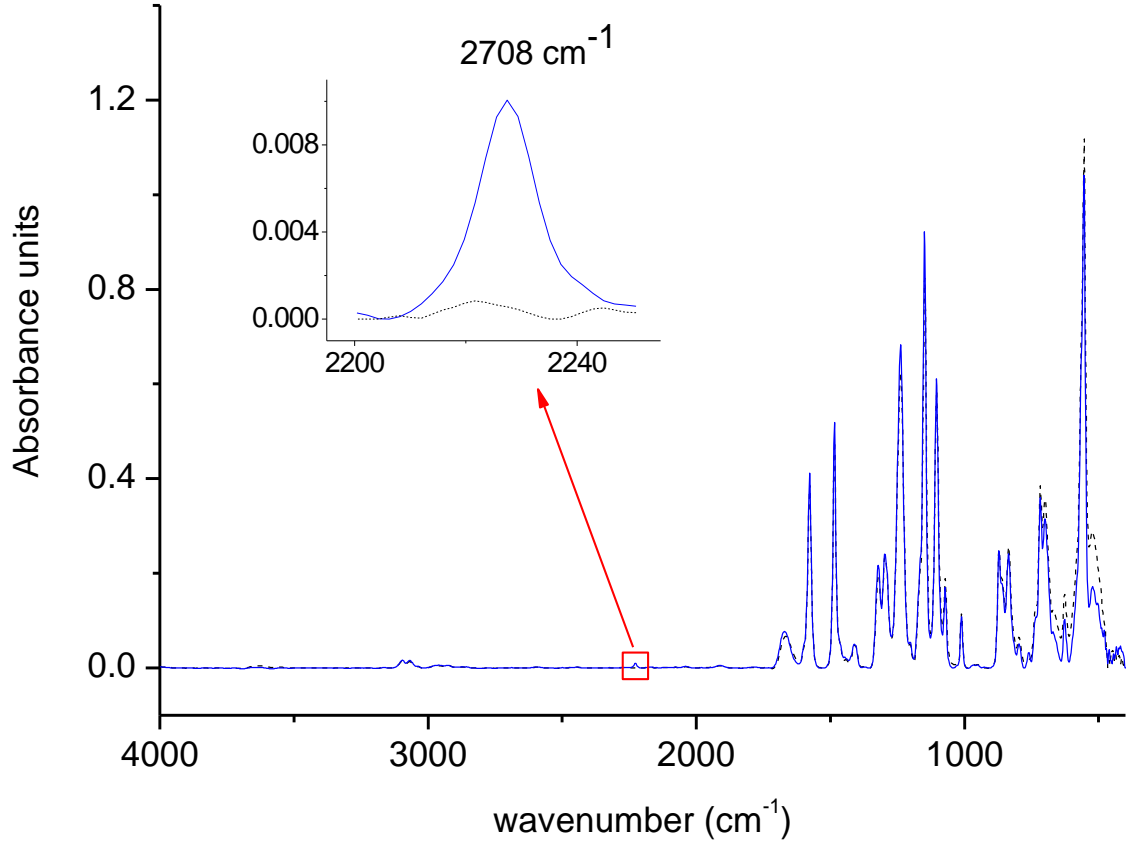

(a)

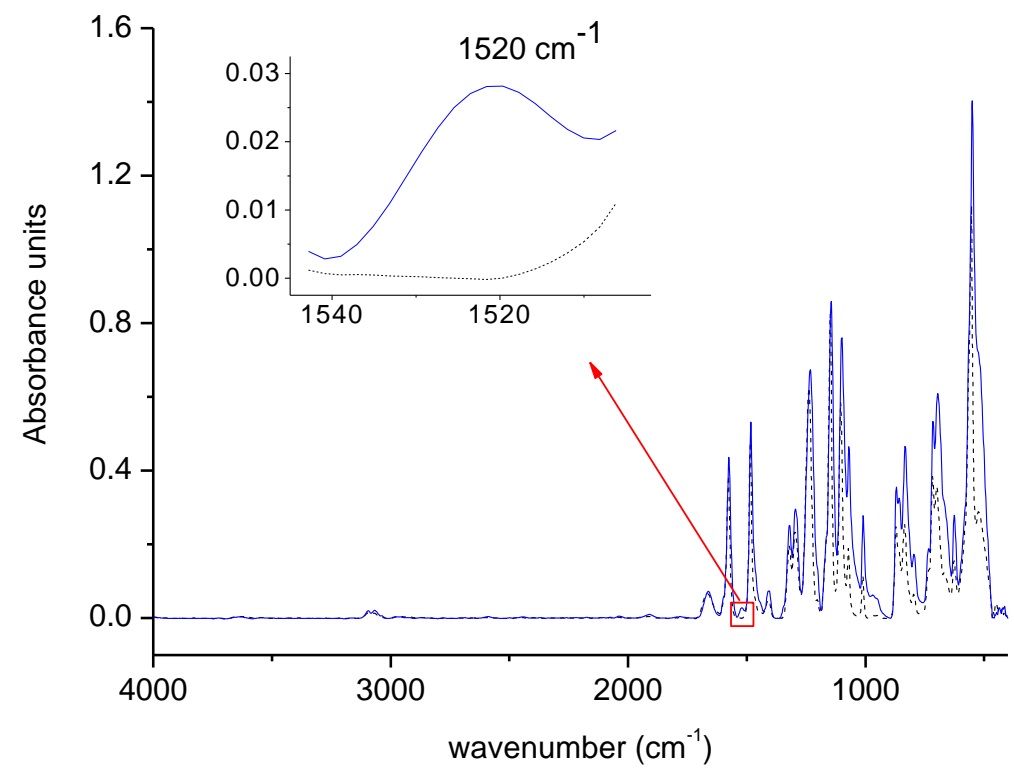

(b) 


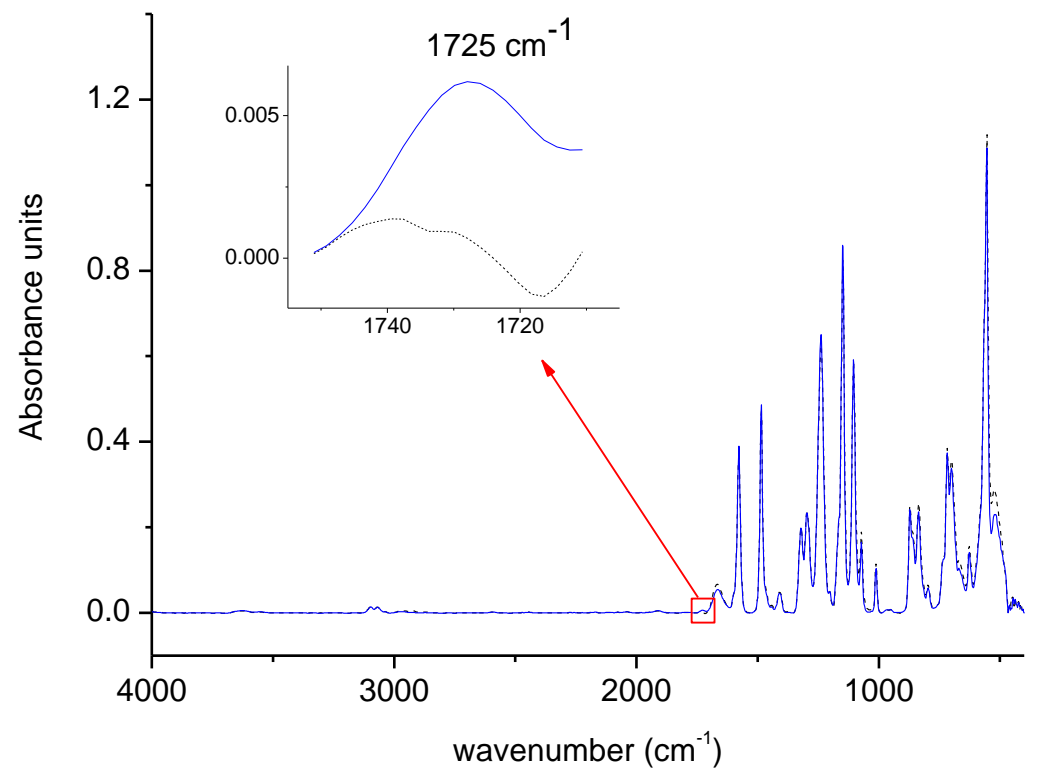

(c)

Figure 4: Representative ATR-FTIR spectra of PES membranes modified (blue solid lines) with solutions of with $3-\mathbf{N}_{2}{ }^{+}$(a), $\mathbf{2}-\mathbf{N}_{\mathbf{2}}{ }^{+}$(b) and $\mathbf{4 - \mathbf { N } _ { 2 }}{ }^{+}$(c). Unmodified PES membrane spectra are also represented with black dashed lines. Blow-ups of the spectra are shown in insets. 


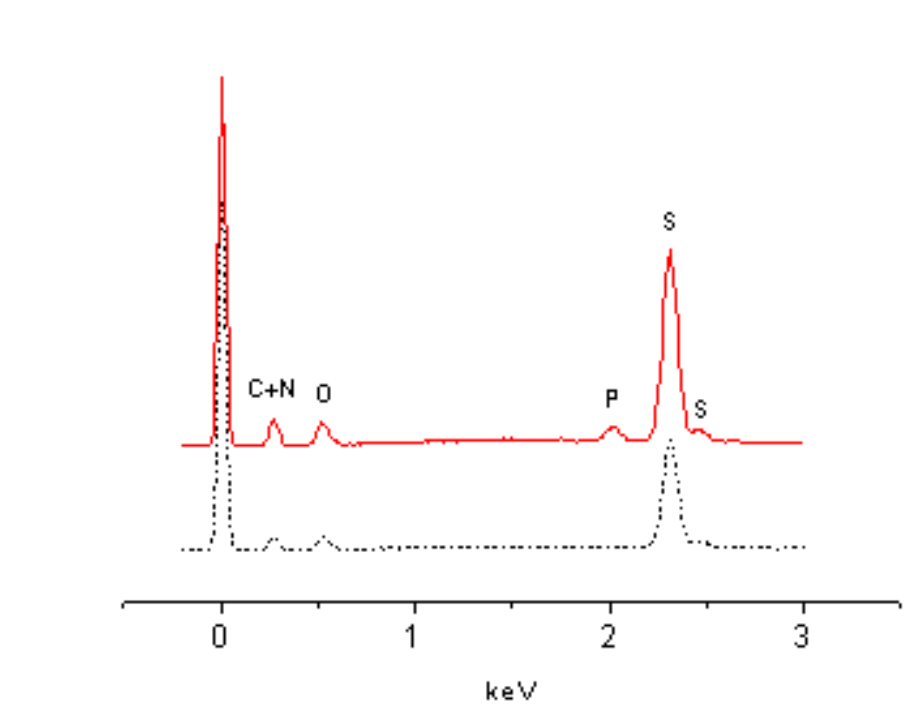

Figure 5: EDX spectra of PES membranes before (black dashed line) and after (red solid line) modification by $\mathbf{1}-\mathbf{N}_{2}{ }^{+}$. 


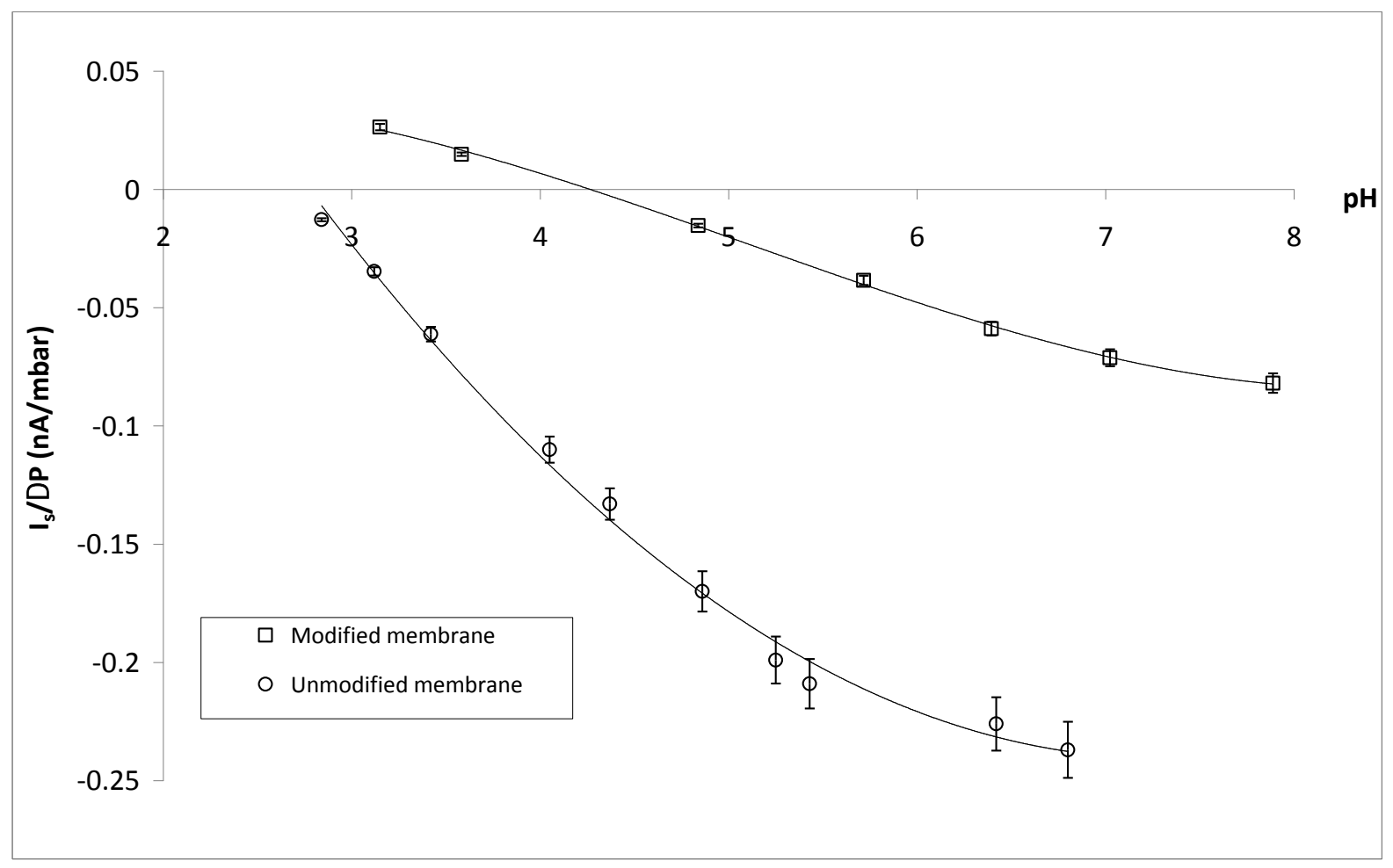

Figure 6: $\mathrm{pH}$ dependence of the streaming current coefficient $\left(\mathrm{I}_{\mathrm{S}} / \Delta \mathrm{P}\right)$ measured with different membranes; circles: unmodified PES membrane; Squares: PES membrane modified by $\mathbf{1}-\mathbf{N}_{\mathbf{2}}{ }^{+}$. 


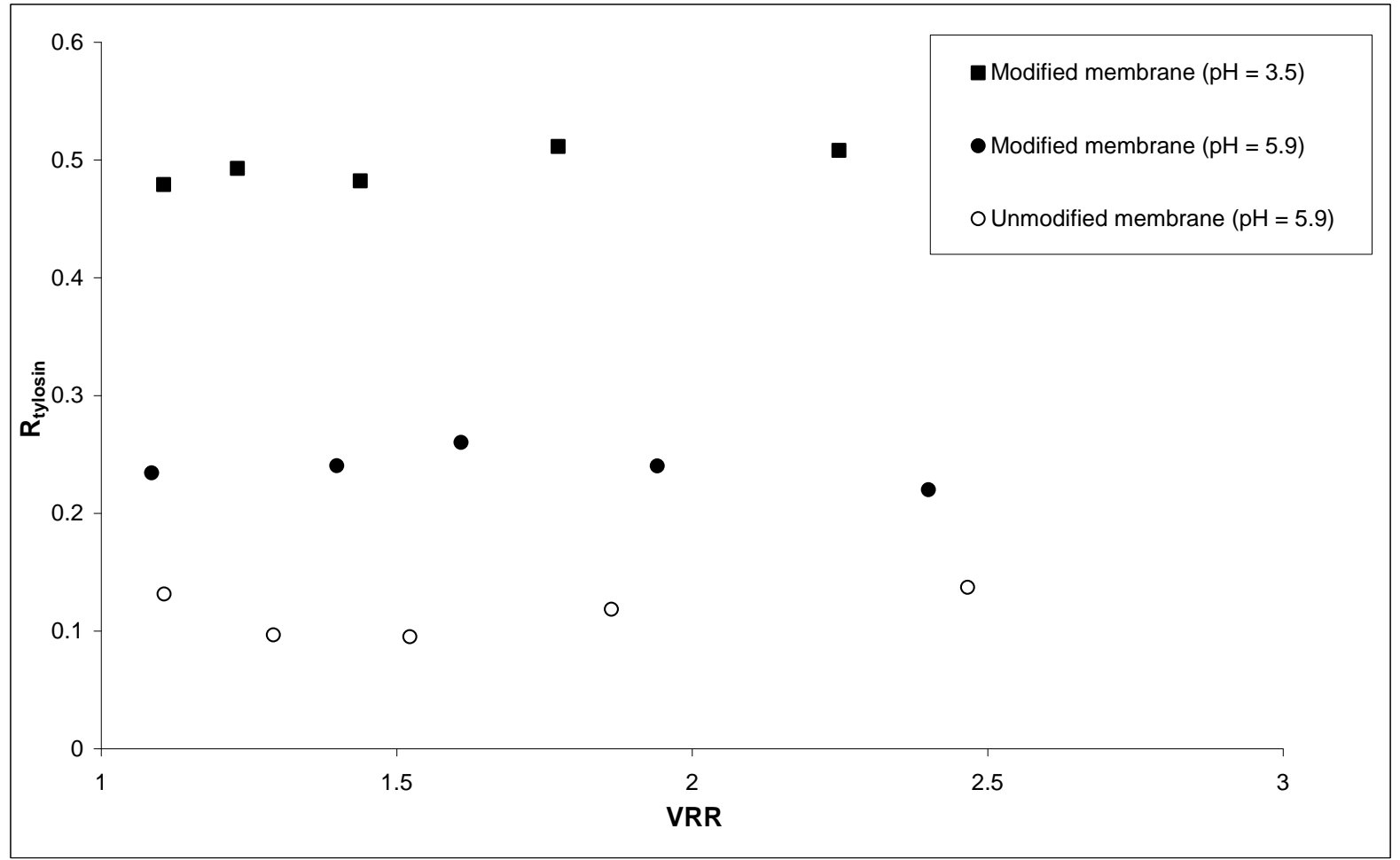

Figure 7: Rejection rate of tylosin as a function of the volume reduction factor (VRR) for the unmodified PES membrane (open symbols) and the membrane modified by $\mathbf{1}-\mathbf{N}_{\mathbf{2}}{ }^{+}$ (closed symbols). 


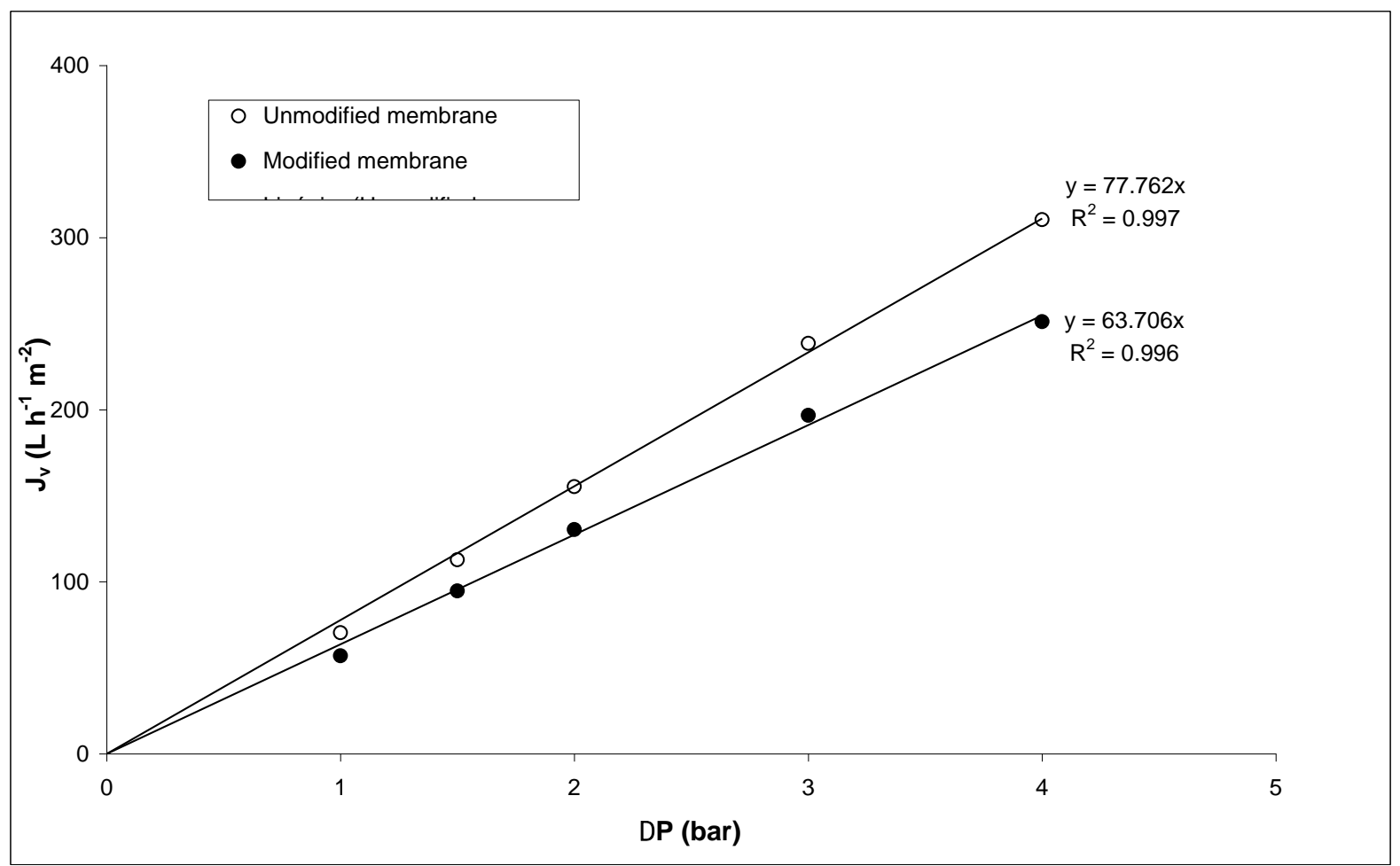

Figure 8: Pure water flux of unmodified and modified (by $\mathbf{1}-\mathbf{N}_{\mathbf{2}}^{+}$) membranes against transmembrane pressure difference. 